\title{
Sarcomatoid Carcinoma of the Penis: An Uncommon Penile Neoplasm
}

\author{
Sucheta Gandhe ${ }^{1}$, Rahul Patil ${ }^{1}$, Raj Nagarkar ${ }^{2}$
}

1. Department of Pathology, HCG Manavata Cancer Centre, Nashik, Maharashtra, India

2. Department of Surgical Oncology, HCG Manavata Cancer Centre, Nashik, Maharashtra, India

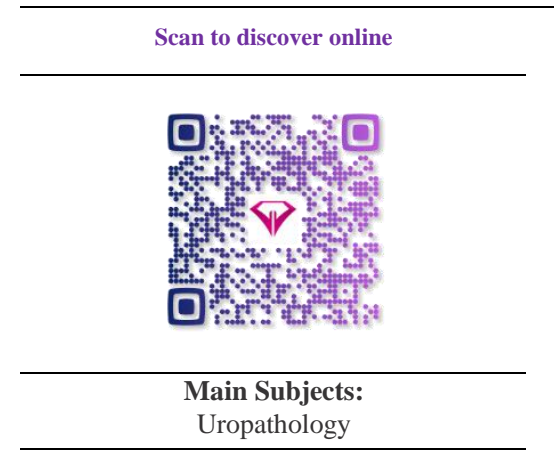

Received 22 Nov 2019;

Accepted 09 Feb 2020;

Published Online 28 Feb 2020;

\section{ABSTRACT}

\begin{abstract}
Sarcomatoid squamous cell carcinomas are extremely rare, high grade, aggressive variant of penile cancers. Sarcomatoid carcinoma are biphasic neoplasms with a combination of both sarcomatoid components and carcinomatous elements. These neoplasms are very rare in the urogenital system. We report a 53-year-old male presented with an ulcerated lesion on the glans penis. The rarity of this case reiterates the importance of thorough morphological and histological examination along with immunohistochemistry in diagnosing, staging, treatment and follow up of patients.
\end{abstract}

KEYWORDS: Immunohistochemistry, Penis, Sarcomatoid carcinoma

$\underline{10.30699 / i j p .2020 .117401 .2275}$

Corresponding Information: $\quad \begin{aligned} & \text { Sucheta Gandhe, Department of Pathology, HCG Manavata Cancer Centre, Nashik, Maharashtra, India. Email: } \\ & \text { academics@manavatacancercentre.com }\end{aligned}$

Copyright ( $\odot$ 2020. This is an open-access article distributed under the terms of the Creative Commons Attribution- 4.0 International License which permits Share, copy and redistribution of the material in any medium or format or adapt, remix, transform, and build upon the material for any purpose, even commercially.

\section{Introduction}

Penile cancer is a rare neoplasm with an annual incidence varying from 0.2 to 1 per $1,00,000$ men, worldwide annually (1). Among all penile cancers, $95 \%$ of the cases histologically correspond to squamous cell carcinomas (SCCs). Remaining 5\% are sarcomatoid carcinomas (SC), which are extremely aggressive and rare form of penile cancers. SC patient's age widely ranges from 28 to 83 years and the glans penis is the most frequently affected area. Only 40 cases of sarcomatoid penile carcinoma have been reported worldwide to date $(2,3)$.

$\mathrm{SC}$ has also been called metaplastic, spindle cell, or biphasic SCC. Most pathologists today accept that SC as a tumor, originates from an epithelial cell. They also express both mesenchymal and epithelial antigens when tested by immunohistochemistry (IHC) $(4,5)$.

Here we are reporting a patient with penile SC treated at our institute (HCG Manavata cancer centre, India) with the necessary clinicopathologic correlations. It's a clinically significant case because of its aggressiveness, rarity, clinicopathologic curiosity and lower survival rate.

\section{Case Report}

A 53-year-old male was presented to our hospital with an ulcerated lesion on the glans penis over past 3 months. A $4 \times 3.5 \mathrm{~cm}$ ulcerated neoplasm involving the dorsal aspect of the glans penis was revealed on clinical examination. The urethral meatus and penile shaft are free from neoplasms. There were significantly enlarged nodes approximately measuring $4 \times 4 \mathrm{~cm}$ in the left inguinal region.

A CT revealed heterogeneously enhancing lesion measuring $3.9 \times 3.7 \times 4.1 \mathrm{~cm}$ in glans penis contiguous distal end of corpora cavernosa and spongiosum. Multiple enlarged lymph nodes were observed in the bilateral external iliac and inguinal region with the largest measuring $3.2 \times 3.1 \mathrm{~cm}$ in the left inguinal region.

The patient underwent a partial amputation with groin node dissection in May 2018. The patient had an uneventful postoperative recovery.

Gross features such as ulcero-proliferative growth was observed on the glans penis (Figure 1A). Histological hematoxylin and eosin (H\&E) stained sections has shown features of a high-grade pleomorphic spindle cell neoplasm with high mitotic activity (15 to 20 mitosis/10 HPF). The tumor cells were arranged in interlacing fascicles with moderate eosinophilic cytoplasm. Occasional areas of atypical squamous epithelial cells were observed (Figure 1B). Histopathologic (HP) reports have shown tumor as a poorly differentiated carcinoma with nodal involvement.

Immunohistochemistry (IHC) showed positivity for P63 (Figure 1C), P40 and Vimentin (Figure 1D). The tumor cells were negative for desmin, HMB45, and smooth muscle actin.

Based on the IHC and HP findings, a diagnosis of SC of the penis was made. 
The patient received CTRT 50 Gray in 25 fractions with concurrent cisplatin (5 cycles), post-operative till July 2018. After 10 months of regular follow-up, CT Pelvis was done which revealed bilateral external iliac and left para-aortic lymphadenopathy. The biopsy of the iliac node revealed metastatic SC in a known case. Bilateral retroperitoneal lymph node dissection was done in May 2019. The patient received an additional three cycles of gemcitabine and carboplatin.

Based on the initial diagnosis, the best possible treatment was selected and initiated as per clinical practice guidelines laid by the National Comprehensive Cancer Network (NCCN). The patient is currently doing well and due for follow-up.

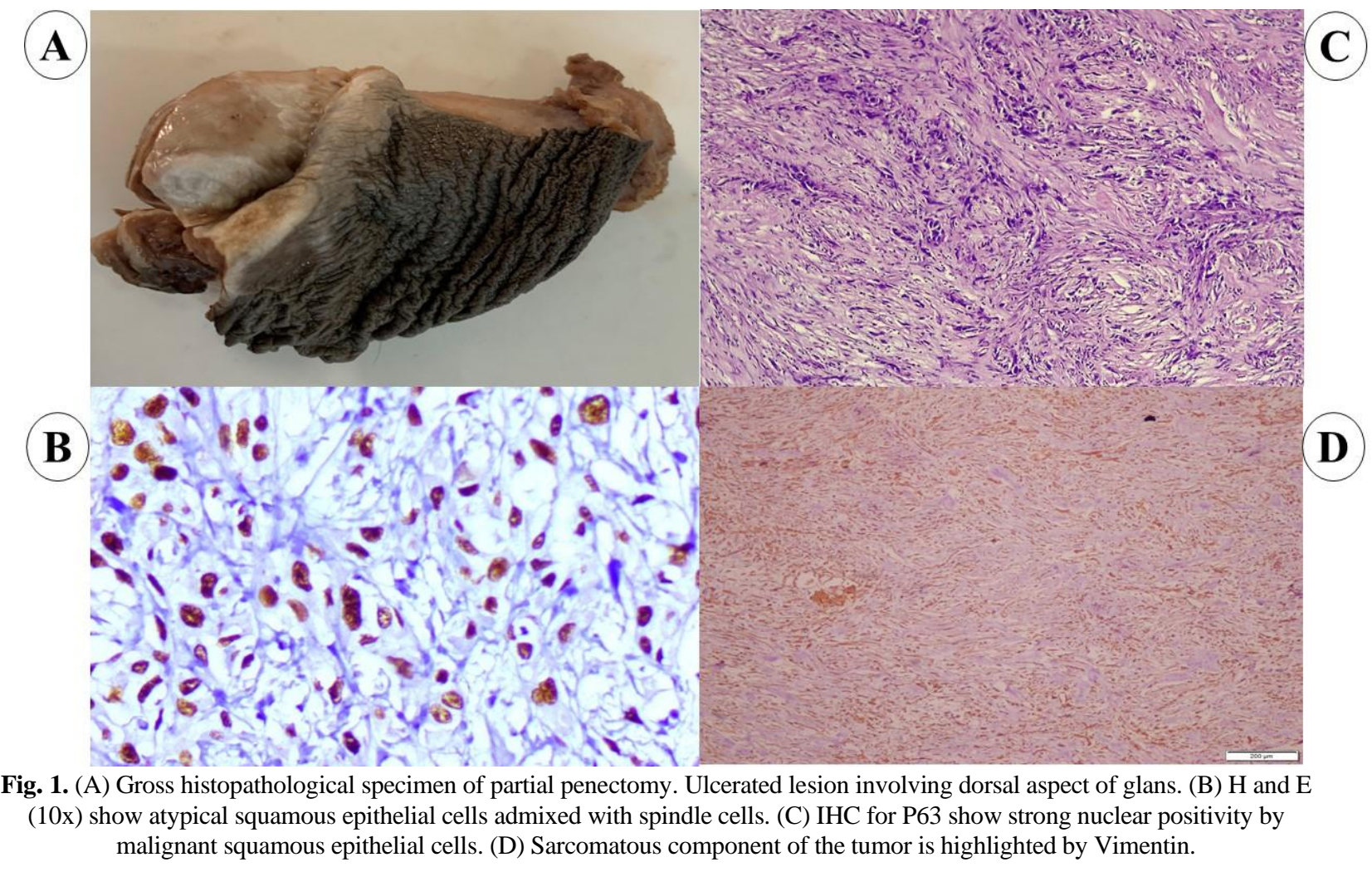

\section{Discussion}

$\mathrm{SC}$ is an unusual, large and aggressive tumor associated with a higher rate of lymph node metastasis and poor prognosis (6). Lymph node or distant metastasis was reported in many cases, suggesting that marked vascular infiltration is a cause of the poor prognosis. Apart from direct hematogenous and lymphatic spread, "Satellitosis" is another mode of metastasis, which is commonly observed in patients with SC, and high-grade SCCs $(7,8)$.

The penile neoplasm morphological features are similar to their counterparts in other locations, with higher number of malignant spindle cells and lower number of squamous cell components as in our case. Sarcomatoid carcinoma are biphasic neoplasms with a combination of both sarcomatoid components and carcinomatous elements (1). As they have both epithelial and mesenchymal components, dissemination occurs via the hematogenous and lymphatic systems leading to both regional and distant metastasis (5).

Positive prognostic factors can always be correlated with the overall survival of the patients. The patient's prognosis depends on various prognostic factors such as size, nuclear grade, nodal metastasis, tumor stage, and chemokine receptors expression (i.e., CXCR2 and CXCR3), etc. $(1,3,5,9)$. Positive prognostic factors for tumorigenesis in $\mathrm{SC}$ of the penis can be correlated with similarities observed in other SC cases i.e., renal cell carcinoma (9). An accurate, thorough morphological, $\mathrm{HP}$, and IHC examination is highly recommended in diagnosing, staging, treatment and follow-up of patients.

Risk factors for SC of penis include human papillomavirus (HPV) infection, poor hygiene, and phimosis. Intratumoral vascular proliferation, lymphovascular emboli, perineural invasion, and overexpression of chemokine receptors may cause metastasis (10). Bijan et al. observe the clinicopathologic factors of patients and prognostic value of the expression of CXCR2 and CXCR3 markers in SC of renal cases. Results from their study have correlated and concluded that higher expression of CXCR3 and CXCR3 was observed with disease progression, which in turn lead to shorter overall survival (9).

In a retrospective study conducted by Lont et al. in 2004, the incidence of SC of the penis (1.4\%) was 
observed to be very rare ( 5 cases, over a span of 46 years), and they have studied all its related IHC, morphological, and clinical features over the years in all the reported cases (5). In another study, Velazquez et al. had defined and evaluated the clinicopathological features of $15 \mathrm{SC}$ cases (4\%) on a retrospective analysis of 400 cases of squamous cell carcinoma of the penis (3). Many patients were presented with either focal or distant metastasis, with a mortality rate of $50-80 \%$ $(3,5)$. In the present case, our treatment protocol goes along with previously conducted studies. Overall our findings from the case are in agreement with the previously reported studies $(1,2,3,5,8)$. Early diagnosis and treatment is the only coping method. Palliative surgery may be considered in patients with ulcerated advanced tumors, which will help them in providing temporary relief from pain, bleeding and tumor regression over a period of time (1).

\section{Conclusion}

$\mathrm{SC}$ of the penis is an unusual entity while the mainstay of treatment remains surgery. SC of the penis is associated with poor prognosis. SC should be differentiated from poorly differentiated squamous carcinoma, melanoma, and sarcoma. An accurate, thorough morphological and histological examination along with IHC would help in diagnosing this rare aggressive entity.

\section{Acknowledgements}

We would like to thank Dr. Yasam Venkata Ramesh from HCG Manavata Cancer Centre, Centre of Difficult Cancers, Nashik, India, for his medical writing assistance.

\section{Conflict of Interest}

The authors declared that there is no conflict of interest regarding the publication of this article.

\section{References}

1. Shankar K, Kumar MV, Srinivas C, Nayak S, Suma MN. Sarcomatoid Carcinoma of the Penis. Indian J Surg Oncol . 2017;8(1):85-7. [DOI:10.1007/s13193-0160531-3] [PMID] [PMCID]

2. Challa VR, Swamyvelu K, Amirtham U, Shivappa P. Sarcomatoid Carcinoma of Penis with Bilateral Inguinal Metastases - A Case Report and Review of Literature. Indian J Surg. 2014;76(4):316-8. [DOI:10.1007/s12262-013-0965-6] [PMID] [PMCID]
3. Velazquez EF, Melamed J, Barreto JE, Aguero F, Cubilla AL. Sarcomatoid carcinoma of the penis: A clinicopathologic study of 15 cases. Am J Surg Pathol. 2005;29(9):1152-8. [DOI:10.1097/01.pas.0000160440.46394.a8] [PMID]

4. Reuter VE. Sarcomatous lesions of the urogenital tract. Semin Diagn Pathol. 1993;10(2):188-201.

5. Lont AP, Galle MPW, Snijders P, Horenblas S. Sarcomatoid squamous cell carcinoma of the penis: a clinical and pathological study of 5 cases. J Urol. 2004;172(3):932-5.

[Doi:

10.1097/01.ju.0000136363.90911.e5] [DOI:10.1097/01.ju.0000136363.90911.e5] [PMID]

6. Cubilla AL, Reuter V, Velazquez E, Piris A, Saito S, Young RH. Histologic classification of penile carcinoma and its relation to outcome in 61 patients with primary resection. Int J Surg Pathol. 2001;9(2):111-20. [DOI:10.1177/106689690100900204] [PMID]

7. Velazquez EF, Soskin A, Bock A, Codas R, Barreto JE, Cubilla AL. Positive Resection Margins in Partial Penectomies: Sites of Involvement and Proposal of Local Routes of Spread of Penile Squamous Cell Carcinoma. Am J Surg Pathol. 2004;28(3):384-9. [DOI:10.1097/00000478-200403000-00012] [PMID]

8. Chandna A, Parmar K, Gupta K, Balamurugan T Sarcomatoid Cancer of Penis in a 45 Year Old Male. Urology, 2019;125:20-3. [DOI:10.1016/j.urology.2018.11.027] [PMID]

9. Bijan R, Banafshe D, Hamid P, Emad Y, Babak M, Katayoun Z, et al. Immunohistochemical Distinction of Metastases of Renal Cell Carcinoma with Molecular Analysis of Over expression of the Chemokines CXCR2 and CXCR3 as Independent Positive Prognostic Factors for the Tumorigenesis. IUBMB life. 2016;68(8):629-33. [DOI:10.1002/iub.1520] [PMID]

10. Shabbir M, Barod R, Hegarty PK, Minhas S. Primary prevention and vaccination for penile cancer. Ther Adv Urol. 2013;5(3):161-9 [DOI:10.1177/1756287212465456] [PMID] [PMCID]

11. Koujalagi RS, Uppin SM, Togale MD, Chetan JV. Sarcomatoid Carcinoma of Penis: A Rare Penile Neoplasm. Journal of Evidence based Medicine and Healthcare. 2014;1(11):1374-7. [DOI:10.18410/jebmh/2014/204] 